\title{
Analysis of Genetic Characteristics of Placenta Mesenchymal Stem Cells Cultured In Vitro Placental
}

\author{
Jianhua $\mathrm{Gao}^{1}$, Hua Cao ${ }^{2}$, Yanhui Wang ${ }^{3}$ \\ ${ }^{1}$ Jiangxi Medical College, Shangrao Jiangxi, 334000 \\ 59624917@163.com
}

\section{Keywords: Genetic Characteristics; Stem Cells; Vitro Placental}

\begin{abstract}
In this study, the conventional method to obtain human placenta derived mesenchymal stem cells (Human placentachorion derived MSCs, HCMSCs), on this basis, on conditions and biological characteristics in vitro amplification HCMSCs preliminary analysis, so as to experimental research and clinical applications quickly and efficiently provide seed cells foundation.
\end{abstract}

\section{Introduction}

Mesenchymal stem cells are a class of self-proliferation and differentiation potential of pluripotent stem cells, stem cells in recent years has become a hot research field, which not only supports the hematopoietic system, but also to mesoderm and ectoderm origin under the specific culture conditions tissue differentiation, can differentiate into bone, fat, cartilage, muscle, endothelial and nerve mesodermal, ectodermal cells derived, MSCs have in vitro inhibition of lymphocyte proliferation in vivo increase in skin graft survival of unique immunological features in the long-term survival in allogeneic environment advantage, while MSCs have self-renewal capacity, in recent years, studies have shown that transplantation, MSCs transplantation not only have the ability to prevent immune rejection, but also has immunomodulatory effect, therefore, the MSCs used in clinical treatment becomes possible.

Currently, the study focused on the source of MSCs in the bone marrow, and its advantages are isolated and cultured in vitro method is simple, fast growth, but in the adult bone marrow MSCs were very low, about 0.001 to $0.01 \%$ of nucleated cells, and bone marrow MSCs The number and differentiation capacity with age and reduce and weaken. Therefore, finding a rich new source of MSCs and the establishment of a stable culture system while avoiding the ethical constraints are a challenge. As the placenta in pregnant women after pregnancy waste, on the basis of informed consent, and to donate bone marrow or peripheral blood collection compared to mobilization, donor no pain, drawn relatively unrestricted, and the pressure of public opinion against the use of their research. In summary, the establishment of human placental source of MSCs cultured in vitro methods and biological factors involved, which quickly and efficiently obtain MSCs from human placenta as a source of seed cells has important value.

\section{Mesenchymal Stem Cells in Placenta}

The placenta is vital organs between fetal and maternal material exchange, which is the parent organization of the Commonwealth of embryos and, by the amnion, chorion and decidua constitute the bottom. The placenta is a round or oval disc structure, divided into sub-surface decent and maternal, child Decent be amnion, umbilical cord is generally attached to its center, umbilical artery, umbilical vein from the umbilical cord was around the point of attachment to the branch when full-term pregnancy radial distribution until edge of the placenta, and then subdivided become villi vessels. Placenta maternal placental surface is divided into 18-20 leaflets. Which is the main part of chorionic placenta, placenta middle, when the blastocyst embedded in the endometrium, the outer covering of the blastocyst trophoblast cells grow into many thin hair, toward the direction of the growth of the uterine wall layer of fluff Because nutrient-rich, vigorous development, constitute the 
main part of the placenta, it all belongs to the fetus, the fetus service, the flowing blood in each of the villi are fetal blood, suggesting a more immature villi, there may be more MSCs .

Zhang Yideng using IMDM containing heparin via umbilical cord loop formation perfusion way to remove the residual cord blood in the placenta, and then placed under sterile conditions placenta medium soaked overnight at room temperature, by density gradient centrifugation from an extract the isolated placental MSCs, and conducted a systematic study of the biological characteristics and functions and so on. Fukuchi such as the use explant tissue block to obtain a leaflet from the mature placenta mesenchymal stem / progenitor cells, stem cells can express several genes and tissue-specific gene signature. In'tAnker etc. amniotic membrane with $10 \mathrm{~cm} 2$ or $10 \mathrm{~cm} 3$ deciduas using medium supplemented with endothelial growth factor and $1 \%$ gelatin-coated culture, successful MSCs from the second trimester and third trimester placenta and amniotic decidua, fetal amniotic MSCs source decidua MSCs is maternal in origin, the overall success rate of over $70 \%$. Yen and other get removed from the umbilical cord blood of mature placental fibroblast-like pluripotent cells. Therefore, MSCs isolated from the placenta is more commonly used method is minced using mechanical and enzymatic digestion, cell suspensions obtained from placental tissue, in order to obtain a cell density gradient centrifugation, or using cell culture substrate with the adhesion of using stickers wall screening method, or marked with the flow cytometric sorting based on cell size and surface.

\section{Human Placenta Mesenchymal Stem Cells Isolated and Cultured}

Mesenchymal stem cell (mesenchymal stem cells, MSCs) is a class of closely watched pluripotent stem cells. Many research groups found, MSCs induced under different conditions can differentiate into osteoblasts, chondrocytes, adipocytes, myoblasts, glial cells. MSCs are capable of self-renewal and differentiation potential, it is suitable seed cells for genetic and degenerative diseases such as cell therapy, has a good prospect in clinical therapy. Previous studies focused on human bone marrow-derived MSCs, but because of human bone marrow-derived MSCs obtained through bone marrow aspiration, and its growth in the number and age declined sharply, limiting the application of human bone marrow-derived MSCs. Therefore, more and more researchers to look for other sources of MSCs, including peripheral blood, umbilical cord blood, umbilical cord, placenta and deciduous. As the placenta in pregnant women after pregnancy waste, its origin is widely and readily available and debate their research and application without ethics, has become a new hotspot MSCs sources. 2007 Source MSCs from human placenta Research International Conference on Human Placenta Derived MSCs clinical application potential and fully affirmed. However, the distribution of MSCs in placenta and how to effectively obtain and expanded in vitro are problems to be solved. To this end, the present study first by immunohistochemical staining identified in the placenta tissue localization of MSCs, and using conventional methods in vitro HCMSCs, and biological characteristics of the amplified HCMSCs preliminary analysis.

After selecting placental tissue to be cut to $1 \times 1 \times 0.5 \mathrm{~mm} 3$ size tissue blocks, fixed, gradient alcohol dehydration, xylene, wax, embedding, sectioning, wax after antigen retrieval, sections were placed in a buffer pressure cooker boil, then paraffin method and then SP immunohistochemical staining. Specific methods are as follows: 0.03\% H2O2- methanol for $30 \mathrm{~min}$, a solution of normal goat serum, incubated at room temperature for 30 minutes, do not wash, dropping antibody (anti-human CD105 antibody dilution of 1: 100, an anti-human CD90 antibody dilution 1:50), with PBS instead of primary antibody as a negative control, $37{ }^{\circ} \mathrm{C}$ incubated for two hours. PBS wash, a solution that is universal secondary antibodies used were incubated $37{ }^{\circ} \mathrm{C} 30 \mathrm{M}$, PBS washed three times, DAB-H2O2 color, specimen dehydration, xylene, neutral resin were mounted, observed under the microscope and photographed.

Select cesarean term placenta, clipping placenta, PBS repeated washing, after the removal of blood, the placenta finely cut into small pieces (ordinary glass pipette can freely draw prevail), $0.1 \%$ IV collagenase at $37^{\circ} \mathrm{C} 15$ minutes, L-DMEM containing $10 \%$ fetal bovine serum. After the reaction was terminated by pipetting repeatedly, over a 100-mesh sieve, the obtained cell 
suspension was centrifuged 1000rpm for 10 minutes, the supernatant was discarded, the use of different culture conditioned medium containing cytokines, set at $37{ }^{\circ} \mathrm{C}, 5 \% \mathrm{CO} 2$ incubation chamber culture medium was changed every 3 Vincent once passaged cells by conventional methods.

A mixture of $10 \%$ FBS L-DMEM culture medium after cell passage 2, after $0.25 \%$ trypsin /0.01\%EDTA digested with PBS containing 1\% fetal calf serum was adjusted to $5 \times 105 / 50 \mu \mathrm{l}$ After then were added to mouse anti-human monoclonal antibody CD73-PE, CD90-PE, CD105-PE, CD14-PE, CD34-FITC, CD45-PE, HLA-DR-PE, at $4{ }^{\circ} \mathrm{C}$ refrigerator for 20 minutes at washed twice with PBS, flow cytometry cell phenotype.

\section{Cytokines Promote Proliferation of HCMSCs in Vitro}

Research on mesenchymal stem cells mainly in the bone marrow, but due to the inconvenience drawn influence ethical constraints and other factors, restricted the bone marrow derived mesenchymal stem cells, further research and application. The placenta as the fetus's organs, its inner mesenchymal stem cells express surface markers part of embryonic stem cells, such as SSEA-4 [1], is due to placental source itself cell excellent properties of mesenchymal stem cells, rich placenta-derived and easily obtained and so on, has become a new source of mesenchymal stem cells research focus. Thus, in 2007, between placenta derived mesenchymal stem cell researchers on between human placenta source charge cultured mesenchymal stem cells, identification, differentiation and other issues convened an international conference, and on mesenchymal stem cells were identified between the placenta source, induced differentiation conditions consensus, and the source of human placenta mesenchymal stem cells cultured in vitro conditions did not put forward arguments. Currently mesenchymal stem cells cultured in vitro between human placenta main source of reference bone marrow-derived mesenchymal stem cells, commonly used L-DMEM culture conditions containing 10\% FBS, there are some researchers by adding $5 \mathrm{ng} / \mathrm{ml}$ of b FGF and 10\% FBS-L- DMEM cultured placenta derived mesenchymal stem cells, but added cytokines whether to change between the biological characteristics of placenta-derived mesenchymal stem cells is controversial. It has been found that some hematopoietic growth and related factors such as FL, SCF, M-CSF and IL-6 has the effect of promoting the hematopoietic stem cells and other stromal cells. Therefore, the present study was to further comparative analysis of the impact of each factor on cell proliferation HCMSCs and biological characteristics.

Stem cells can be divided according to the developmental stages of adult stem cells and embryonic stem cells, since that is the source of embryonic stem cell research restrictions and ethical constraints, so that adult stem cells become widespread concern source of stem cells. Since human placenta Yumi Fukuchi et al. [6] for the first time proved to contain mesenchymal stem cells since, stem cell research is rapidly becoming a hot spot. The advantage lies in its reaction as pregnant women after pregnancy waste, easy to obtain and apply their research without ethical constraints, mesenchymal stem cell research in 2007 the International Conference on Human Placenta-source inter-human placenta derived mesenchymal stem cells in cell phenotype, induce differentiation potential clinical value and the corresponding discussion has affirmed the placenta during pregnancy as provide nutrients to the fetus places, among which contains mesenchymal stem cells are more immature, with a portion of the properties of embryonic stem cells. Therefore placenta mesenchymal stem cells isolated and cultured and involved promoting proliferation of biological factor is the issue of concern in recent years.

Currently more common is the use of culture conditions containing $10 \%$ fetal calf serum L-DMEM, there are some researchers refer culture conditions human bone marrow-derived mesenchymal stem cells, adding $5 \mathrm{ng} / \mathrm{ml}$ of b FGF in the media, but there are reports He said b FGF does not change the human placenta mesenchymal stem cell colony formation, on the contrary will change the human placenta source of mesenchymal stem cell morphology and phenotype. Our results show that, compared with the complete medium without cytokine-containing b FGF culture conditions can partially improve the human placenta mesenchymal stem cell proliferation and cell 
morphology does not change the human placenta mesenchymal stem cells and Phenotype. Similarly, our results also show that the hematopoietic stem cell growth factor FL more effectively promote the proliferation of mesenchymal stem cells from human placenta, does not affect the induction of differentiation and cell morphology, and there is no change in human placenta phenotype of mesenchymal stem cells : high expression of CD90, CD105 and CD73, low expression of hematopoietic stem cell marker CD14, among CD34, CD45 and HLA-DR, the above results and the source of international conferences placental mesenchymal stem cells phenotype standards. Then complete medium containing FL vitro amplification of HCMSCs puts into neuron-like cells, adipocyte differentiation results show that with complete medium without cytokine HCMSCs complete medium containing FL, amplified and containing bFGF in complete medium cultured cells, have good differentiation potential. Meanwhile, RT-PCR results showed that in vitro amplification of human placental mesenchymal stem cells express a receptor FL flt3, lay the foundation for further research on the mechanism of action of FL. However, all have pro-proliferative effect of cytokines HCMSCs FL and bFGF whether a synergistic effect on the proliferation pending further study.

\section{Conclusion}

The study found a way to effectively acquire and promotion of human placenta derived mesenchymal stem cells in vitro amplification methods, and found that FL has a significant role to promote HCMSCs proliferation and its effect was better than bFGF, which will quickly get the short amount of human placenta mesenchymal stem cells provide a new way, with good use of value.

\section{References}

[1] Jiang Y, Jahagirdar BN, Reihardt RL, Et Al. Pluripotency of Mesenchymal Stem Cells Derived from Adult Marrow. Nature, 2002; 418: 41-49.

[2] Pittenger MF, Mackay AM, Beck SC, Et Al. Multilineage Potential of Adult Humanmesenchymal Stem Cells. Science, 1999; 284:143-147.

[3] Rao MS, Mattson MP. Stem Cells and Aging: Expanding The Possibilities. Mechageing Dev, 2001;122:713-734.

[4] Fernandez M, Simon V, Herrera G, Et Al. Detection of Stromal Cells in Peripheralblood Progenitor Cell Collections from Breast Cancer Patients. Bone Marrow Transplant, 1997; 20: $265-271$.

[5] Erices A, Conget P, Minguell JJ. Mesenchymal Progenitor Cells in Human Umbilicalcord Blood. Br J Haematol, 2000;109:235-242. 